\title{
Interpolating Subdivision for Meshes with Arbitrary Topology
}

\author{
Denis Zorin ${ }^{\dagger}$ \\ Peter Schröder ${ }^{\dagger}$ \\ Wim Sweldens*
}

\begin{abstract}
Subdivision is a powerful paradigm for the generation of surfaces of arbitrary topology. Given an initial triangular mesh the goal is to produce a smooth and visually pleasing surface whose shape is controlled by the initial mesh. Of particular interest are interpolating schemes since they match the original data exactly, and play an important role in fast multiresolution and wavelet techniques. Dyn, Gregory, and Levin introduced the Butterfly scheme, which yields $C^{1}$ surfaces in the topologically regular setting. Unfortunately it exhibits undesirable artifacts in the case of an irregular topology. We examine these failures and derive an improved scheme, which retains the simplicity of the Butterfly scheme, is interpolating, and results in smoother surfaces.
\end{abstract}

\section{Introduction}

Beginning with an initial mesh, subdivision produces a sequence of refinements. New vertices are defined as local affine combinations of nearby vertices. An attractive feature of these schemes is locality, i.e., no global system of equations needs to be solved. Examples of subdivision include classical spline constructions which are generally not interpolating. The most common interpolating scheme is based on piecewise linears. Unfortunately this is not smooth enough for many applications. A scheme that achieves $C^{1}$ continuity in the topologically regular setting, was pioneered by Dyn, Gregory, and Levin $[9,10]$ and has been applied to the construction of smooth surfaces ${ }^{1}$.

The mathematical analysis of the surfaces resulting from subdivision is not always straightforward (see for example Reif [20]). However, the simplicity of the algorithms and associated data structures makes subdivision attractive for large data sets and interactive applications.

Recently, interpolating subdivision has been used for multiresolution analysis of complex geometries [16]. It provides a powerful tool for the constructions of finite analysis and synthesis filters for wavelet algorithms on general manifolds [22] using the lifting scheme[23]. Further, adaptive subdivision is greatly simplified with interpolating rules. Multiresolution decomposition algorithms are of importance in compression, progressive display and transmission, multiresolution editing, and multigrid/wavelet based numerical methods.

While the Butterfly scheme of Dyn, Gregory and Levin can be used to generate smooth surfaces over regular triangular meshes (all vertices have valence 6), it exhibits degeneracies when applied

\footnotetext{
${ }^{\dagger}$ Department of Computer Science, California Institute of Technology, Pasadena CA 91125.

* Lucent Technologies (formerly AT\&T), Bell Laboratories, 700 Mountain Avenue, Rm. 2C-175, Murray Hill, NJ 07974; Department of Computer Science, K.U.Leuven, Belgium. (On leave from the NFWO) dzorin@gg.caltech.edu, ps@cs.caltech.edu, wim@bell-labs.com

${ }^{1}$ An alternative was recently proposed by Kobbelt [13].
}

in a topologically irregular setting: undesirable creases can result at vertices of valence other than 6 . Figure 1 demonstrates such a failure for vertices of valence 3 . The left picture shows the result of applying the original Butterfly scheme to a tetrahedron, the right picture is obtained using our modified scheme.

Motivated by these observations, we consider the construction of a subdivision scheme under the following constraints:

- Interpolation: The original mesh vertices are interpolated and all newly generated vertices are on the limit surface.

- Locality: The neighborhood used to define new vertex positions from old ones should be as small as possible to enable fast algorithms.

- Symmetry: The scheme should exhibit the same type of symmetries as the local mesh topology.

- Generality: The scheme should work for triangulations which are not topologically restricted, including the proper handling of boundaries.

- Smoothness: We require the resulting scheme to reproduce polynomials up to some power-a necessary but not sufficient condition for higher order continuity.

- Simplicity: The scheme should only require simple data structures.

Since the Butterfly scheme satisfies these requirements except for topological generality, we make it the starting point of our investigation.

The main result of our work is a simple modification of the Butterfly scheme around vertices of valence not equal to 6 . It combats the cusp like artifacts exhibited by the unmodified scheme in those circumstances. We use Fourier transform techniques [5, 1], which are typically used for analysis of subdivision, for the synthesis of our new interpolating subdivision scheme.

In the next section we briefly review related work. Then we describe the construction of our modified scheme and present the results. We conclude with a discussion and outlook.

\section{Related Work}

We briefly review subdivision algorithms for arbitrary topology control meshes. They come in two flavors: approximating and interpolating.

\subsection{Approximating Subdivision Schemes}

Approximating subdivision schemes for arbitrary topology meshes are typically modifications of spline based schemes. The algorithms of Doo and Sabin [4, 5, 21] and Catmull and Clark [2] are generalizations of quadratic and cubic B-splines respectively. The behavior around extraordinary vertices was analyzed by Doo and
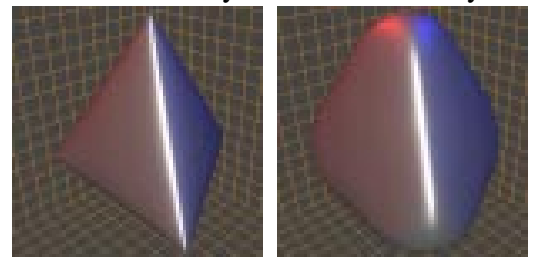

Figure 1: A tetrahedron is subdivided according to the original Butterfly scheme (on the left) and with our modified Butterfly scheme (right). 
Sabin [5] using Fourier transforms and an eigen analysis of the subdivision process. More recently Ball and Storry [1], Warren [24], and Reif [20] refined these techniques. A generalization of quartic box splines for arbitrary triangulations was given by Loop [14].

Alternatively one can directly derive a set of spline patches which globally achieve some order of continuity $[15,18,19,3]$. The output of these algorithms is a set of patches of varying, at times rather high, polynomial order and varying shape.

\subsection{Interpolating Subdivision Schemes}

Since the ability to control the resulting surface exactly is very important in many applications, modifications of approximating schemes have been proposed to force the limit surface to interpolate particular points and normals. Nasri [17] presents such a modification for the Doo-Sabin scheme, while Halstead et al. [11] do the same for the Catmull-Clark scheme. Both cases have a number of limitations. For example, it is unclear under what conditions the linear system to be solved for the interpolation constraints is solvable. Additionally, the interpolation conditions are only satisfied in the limit. Among the patch based schemes only Peters [18] recently gave one which can incorporate interpolation constraints without requiring the solution of a global linear system. We choose a more direct route by considering subdivision schemes which are interpolating by design.

The Butterfly scheme is interpolating, local, and simple to implement, but only leads to $C^{1}$ surfaces in the regular setting [10] (all vertices of the mesh have valence 6.) Topological regularity is a rather severe restriction: the failure to be smooth for vertices of valence other than six can be quite noticeable (see Figures 1 and 4).

\section{Interpolating Subdivision Surfaces}

We first discuss surface smoothness, next present the main idea behind our construction and then give the description of the subdivision algorithm. Space limitations do not permit us to go into the details of the mathematical derivation. These are presented in [25].

\subsection{Smoothness}

One of the advantages of patch based polynomial schemes is that their analytic smoothness properties are well understood and closely correlate with what a human observer would call a smooth surface. This property is only partially covered by such notions as $C^{k}$ continuity, e.g., a $C^{2}$ function may be quite "wiggly." Absence of unnecessary undulations is often referred to as "fairness." For example, Halstead, et al. [11] observed that enforcing interpolation conditions on Catmull-Clark surfaces resulted in a loss of fairness, an issue they addressed with a global optimization pass.

In the present paper we do not consider the question of globally optimal fairness. Nonetheless we are attempting to build smooth interpolating subdivision schemes which yield surfaces whose shape is as pleasing as possible. Because no vertex is ever moved once it is computed, any distortion in the early stages of the subdivision will persist. This makes particularly the first few subdivision steps very important.

Local smoothness of the surface requires the existence of smooth coordinate functions $(x, y, z)(s, t)$. In the next section we discuss the smoothness of coordinate functions in greater detail.

\subsection{The Idea of the Construction}

Consider a single coordinate function. This function can be visualized as the graph of some function over the real parameter plane (not to be confused with the actual surface). More concretely, instead of thinking about generating new vertices in $\mathbf{R}^{3}$ by taking local averages of vertices in the control polyhedron, we are now thinking of scalar values which are assigned to the vertices of a triangulation in $\mathbf{R}^{2}$. This triangulation is refined through splitting each edge in
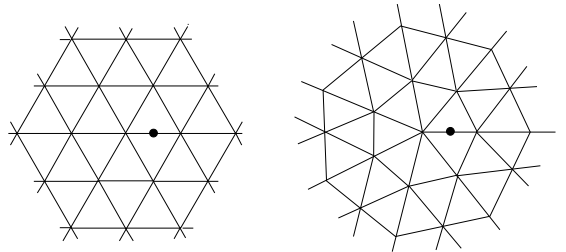

Figure 2: On the left a regular neighborhood in which all vertices have valence 6. On the right an example of a $K=7$ vertex. In both cases the dot represents a midpoint for which we compute a new value. In the regular case the Butterfly stencil is used, while in the case $K \neq 6$ a separate analysis is performed leading to a local modification of the weights.

two and reconnecting. The interpolating subdivision scheme is used to define a value associated with the new point of the planar triangulation by taking suitable weighted sums of nearby values. Note that all new planar vertices will be of valence 6 . The neighbors participating in the computation are part of the subdivision stencil and their weights characterize the scheme.

Since we assume that all our schemes will be local, we need to analyze only a small number of possible cases of the relationship between the new vertex and the topology of its graph neighborhood. The two cases of primary importance are the regular sites (all vertices are regular), and the extraordinary sites (adjacent to a non-regular vertex.) After several subdivision steps, at most one vertex in the neighborhood has valence not equal to 6 , so it is sufficient to analyze behavior of the scheme only on regular and $K$-regular triangulations, with only one extraordinary vertex of valence $K$.

For the regular case, we use the Butterfly scheme which reproduces polynomials of degree 3 . If initial values at the vertices of a regular triangulation are samples of a polynomial function on the plane, the limit of polynomial-reproducing subdivision will be that function.
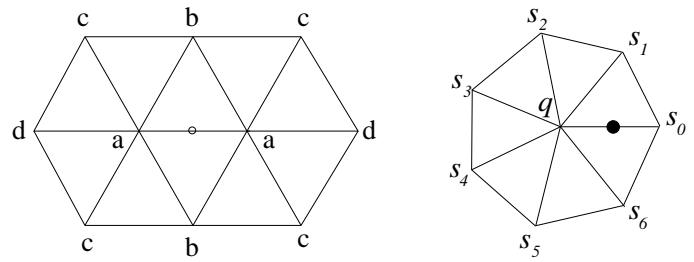

Figure 3: a: Ten-point stencil. The dot indicates the midpoint of the edge for which a new value is computed. $b$ : Stencil for a vertex in the 1-neighborhood of an extraordinary vertex

For the $K$-regular case, we try to choose the coefficients in such a way that the behavior of the scheme is similar to the regular case. To keep the support minimal we use only the immediate neighbors of an extraordinary vertex to compute the values at new sites next to it (see Figure 3(b).) We use a limited version of polynomial reproduction for the neighborhood of an extraordinary vertex: the values of a polynomial function of degree less than 3 have to be reproduced only for the immediate neighbors of the extraordinary vertex on each level, except the case $K=3$, when only some second degree polynomials are reproduced.

The Discrete Fourier Transform and an eigen analysis allow us to to construct subdivision rules with these constraints [25]. The subdivision matrices for extraordinary vertices have the same eigenvalues as the matrices for regular vertices.

\subsection{The Modified Subdivision Scheme}

The subdivision scheme computes a new scalar value for each edge midpoint of the triangulation. We distinguish between four positions of the edge that we subdivide.

1. The edge connects two vertices of valence 6 . In that case we use the extension of the Butterfly scheme to the ten point stencil [8] (see Figure 3a). This is the canonical setting and the weights are 
given by

$$
a=1 / 2-w, b=1 / 8+2 w, c=-1 / 16-w, d=w
$$

where $w$ can be chosen suitably small [8] (we used $w=0$ ).

2 . The edge connects a $K$-vertex $(K \neq 6)$ and a 6 -vertex; the 1 neighbors of the $K$-vertex are used in the stencil as indicated in Figure 3 b. For $K \geq 5$ the weights are given by

$$
s_{j}=(1 / 4+\cos (2 \pi j / K)+1 / 2 \cos (4 \pi j / K)) / K
$$

with $j=0, \ldots, K-1$. For $K=3$ we take $s_{0}=5 / 12, s_{1,2}=$ $-1 / 12$, and for $K=4, s_{0}=3 / 8, s_{2}=-1 / 8, s_{1,3}=0$.

3. The edge connects two extraordinary vertices; in this case we take the average of the values computed using the appropriate scheme of the previous paragraph for each endpoint. Since this case can only occur at the topmost level of subdivision the ultimate smoothness of the scheme is not influenced by this choice. However, we have found that the overall fairness of the resulting shapes tends to be better with this scheme.

4. Boundaryedges are subdivided using the 1-dimensional 4 point scheme $\left(s_{-1}=-1 / 16, s_{0}=9 / 16, s_{1}=9 / 16, s_{2}=-1 / 16\right)[7,6]$. In this case only other edge points participate in the stencil. A consequence of this rule is that two separate meshes, whose boundary is identical, will have a matching boundary curve after subdivision. Edges which are not on the boundary but which have a vertex which is on the boundary are subdivided as before while any vertices in the stencil which would be on the other side of the boundary are replaced with "virtual" vertices. These are constructed on the fly by reflecting vertices across the boundary.

\section{Results}

We built an interactive application supporting general triangular meshes and adaptive subdivision, using restricted triangular quadtrees. All subdivision coefficients are precomputed and stored in a table indexed by the valence $K$ of a given vertex. We present here some results obtained with this application.

In the top half of Figure 4 we show how application of our scheme to an initial polyhedron (on the right) produces a smooth pipe joint. ${ }^{1} \quad$ Note that the original control polyhedron contains vertices of valence 7 and 4 next to each other. Six levels of the original Butterfly subdivision lead to the shape that is shown in the middle. In several regions the surface has creases. In contrast on the top right is the shape resulting from applying our modified scheme to the same original control polyhedron. These images also demonstrate the treatment of boundaries. We applied our scheme to a data set of a mannequin head (courtesy University of Washington) and a torso (a dataset from the Avalon site maintained by Viewpoint Datalab.) The bottom half of Figure 4 shows the original polyhedron and subdivision surfaces approximated with 3 subdivision levels. The mannequin dataset was obtained from the control mesh for the Loop subdivision scheme by moving each control vertex to its limit position on the surface. Then the surface was interpolated using the modified Butterfly scheme.

It is important to note the difference between averaging schemes like Loop's and interpolating schemes. While averaging schemes are able to produce relatively fair surfaces from highly irregular control polyhedra acting as low-pass filters, interpolating schemes by their nature are much more sensitive to the irregularities in the initial mesh. Our examples demonstrate that if the initial mesh is sampled from a smooth surface, the interpolation scheme performs quite well.

\footnotetext{
${ }^{1}$ This configuration was inspired by a similar configuration of Jens Albrecht, Erlangen University.
}

\section{Summary and Future Work}

We have presented a simple interpolating subdivision scheme for meshes with arbitrary topology. Our scheme is based on the Butterfly scheme, with special rules applied in the neighborhood of the extraordinary vertices. The proposed scheme has a number of properties that make it attractive:

- it is interpolating at all levels of subdivision;

- the support of the scheme is minimal;

- it is easy to implement;

- limit surfaces have adequate smoothness;

- subdivision can be performed adaptively;

- explicit formulas for the normals exists [25].

Our scheme is especially convenient for multiresolution representation of surfaces and wavelet representation of functions on surfaces as in [22].

There are several aspects which we believe to be worth investigating further:

- Currently we perform only a limited adaptation of the scheme at the boundary. A more detailed analysis of $K$-vertices on or near the boundary is desirable.

- By collapsing vertices and edges, this scheme can immediately accommodate mesh tagging approaches such as in [12]. Furthermore, the degree of smoothness of the surface can be continuously adjusted by manipulating the remaining degrees of freedom of the scheme.

- Preliminary tests show that Hoppe's subdivision surface fitting methodology works well with the scheme proposed in this paper.

- The question of $C^{1}$ smoothness will be analyzed based on the work of Reif [20] and Warren [24] in a forthcoming paper.

- We have observed that the "fairness" of the surface is determined by the behavior of the scheme at the first two subdivision steps. A more thorough analysis of the scheme in the first subdivision steps may shed more light on how to maintain fairness under the constraint of interpolation.

\section{Acknowledgements}

This work was supported in part by an equipment grant from Hewlett Packard and funds provided to the second author by the Charles Lee Powell Foundation. Additional support was provided by NSF (ASC-89-20219), as part of the NSF/DARPA STC for Computer Graphics and Scientific Visualization. All opinions, findings, conclusions, or recommendations expressed in this document are those of the authors and do not necessarily reflect the views of the sponsoring agencies.

\section{References}

[1] Ball, A. A., and Storry, D. J. T. Conditions for tangent plane continuity over recursively generated B-spline surfaces. ACM Transactions on Graphics 7, 2 (1988), 83-102.

[2] Catmull, E., and Clark, J. Recursively generated B-spline surfaces on arbitrary topological meshes. Computer Aided Design 10, 6 (1978), 350-355.

[3] Dahmen, W., Micchelli, C. A., and Seidel, H.-P. Blossoming begets B-splines bases built better by B-patches. Mathematics of Computation 59, 199 (1992), 97-115.

[4] Doo, D. A subdivision algorithm for smoothing down irregularly shaped polyhedrons. In Proceedings on Interactive Techniques in Computer Aided Design (Bologna, 1978), pp. 157-165.

[5] Doo, D., and Sabin, M. Analysis of the behaviour of recursive division surfaces near extraordinary points. Computer Aided Design 10, 6 (1978), 356-360.

[6] Dubuc, S. Interpolation through an iterative scheme. J. Math. Anal. Appl. 114 (1986), 185-204.

[7] Dyn, N., Gregory, J. A., and Levin, D. A four-point interpolatory subdivision scheme for curve design. Computer Aided Geometric Design 4 (1987), 257-268.

[8] Dyn, N., Hed, S., and Levin, D. Subdivision schemes for surface interpolation. In Workshop in Computational Geometry (1993), A. C. et al., Ed., World Scientific, pp. 97-118.

[9] Dyn, N., Levin, D., and Gregory, J. A. A butterfly subdivision scheme for surface interpolation with tension control. ACM Transactions on Graphics 9, 2 (1990), $160-169$.

[10] Dyn, N., Levin, D., and Micchelli, C. A. Using parameters to increase smoothness of curves and surfaces generated by subdivision. Computer Aided Geometric Design 7 (1990), 129-140.

[11] Halstead, M., Kass, M., and DeRose, T. Efficient, fair interpolation using catmullclark surfaces. In Computer Graphics Proceedings (1993), Annual Conference Series, ACM Siggraph, pp. 35-44. 


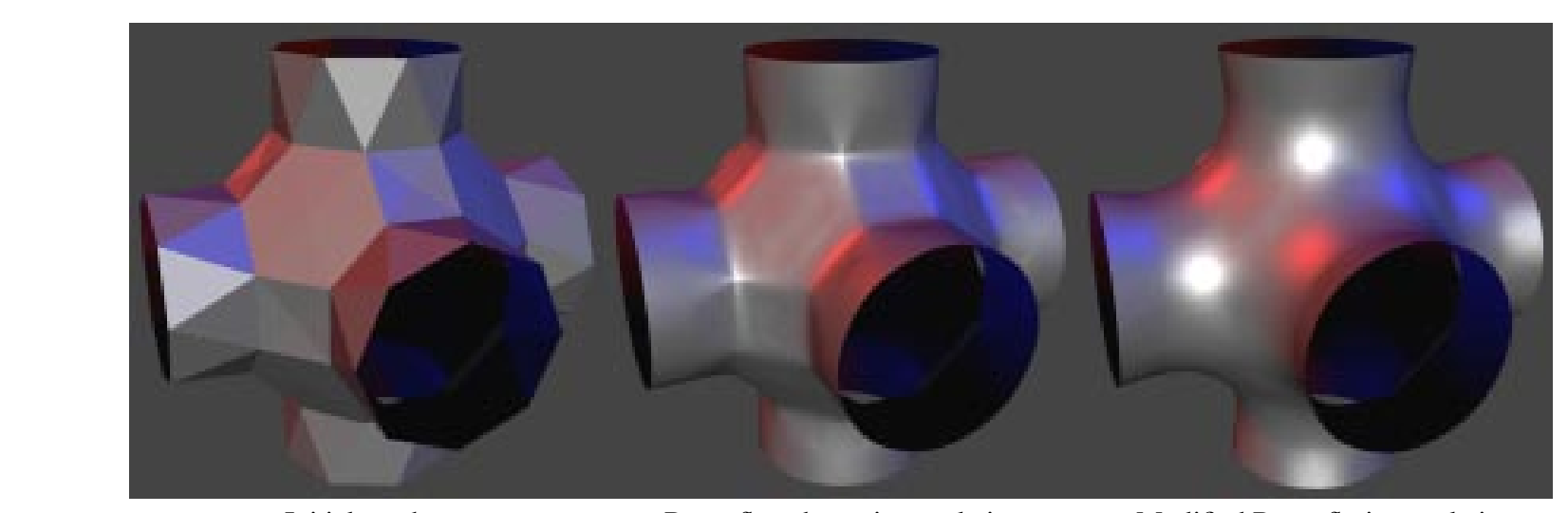

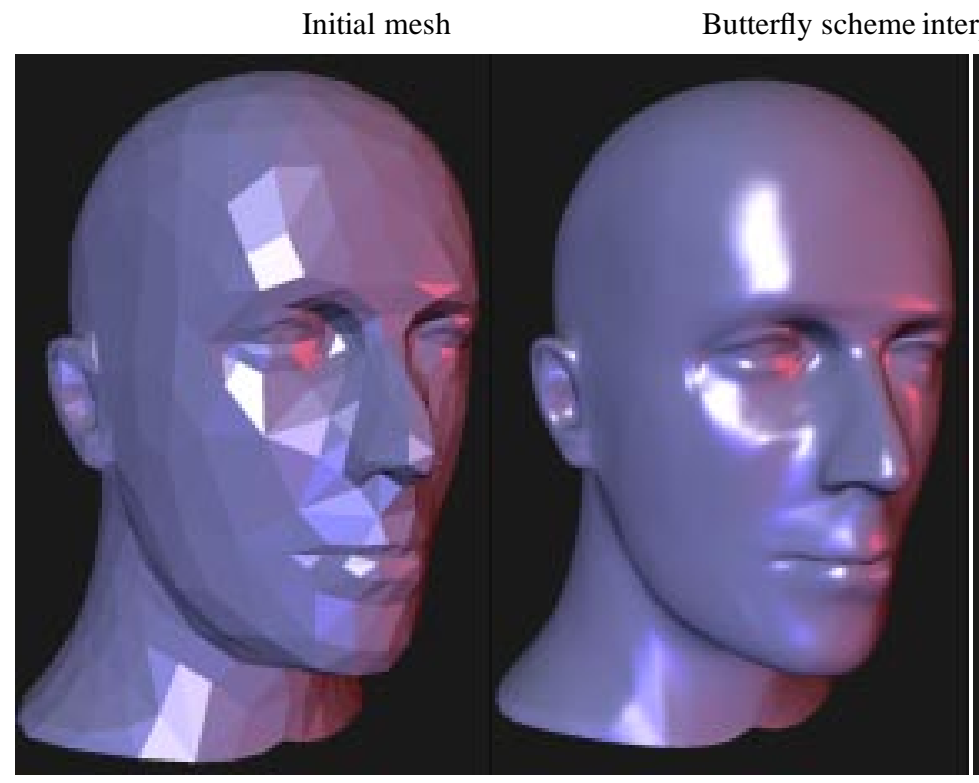

Initial mesh
Modified Butterfly interpolation

\begin{abstract}
rpolation
\end{abstract}

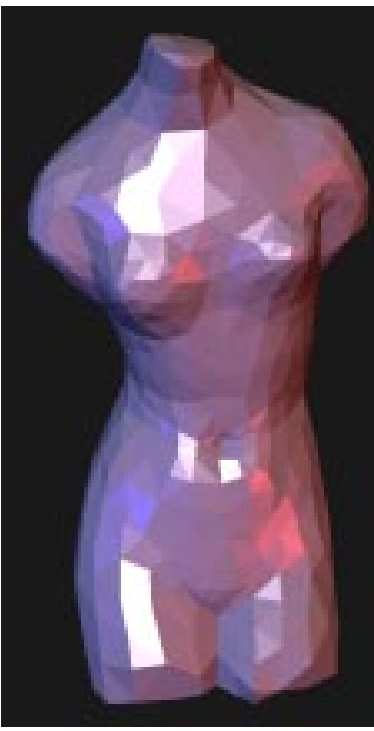

Initial mesh
Modified Butterfly interpolation
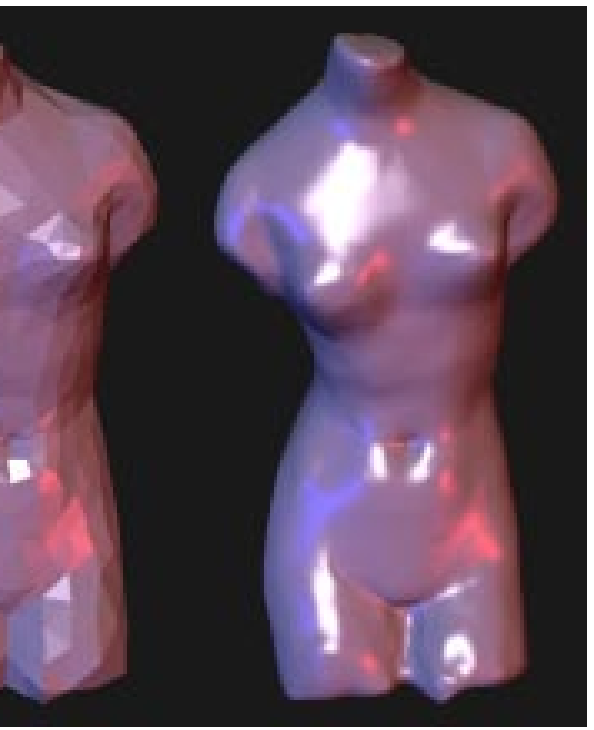

Modified Butterfly interpolation

Figure 4: Top row: pipe joint. Note the difference between Butterfly and Modified Butterfly. Lower left: mannequin head. Lower right: torso.

[12] Hoppe, H., DeRose, T., Duchamp, T., Halstead, M., Jin, H., McDonald, J., Schweitzer, J., and Stuetzle, W. Piecewise smooth surface reconstruction. In Computer Graphics Proceedings (1994), Annual Conference Series, ACM Siggraph, pp. 295-302.

[13] Kobbelt, L. Interpolatory subdivision on open quadrilateral nets with arbitrary topology. In Computer Graphics Forum (1996), vol. 15, Eurographics, Basil Blackwell Ltd. Eurographics ' 96 Conference issue.

[14] Loop, C. Smooth subdivision surfaces based on triangles. Master's thesis, University of Utah, Department of Mathematics, 1987.

[15] Loop, C. Smooth spline surfaces over irregular meshes. In Computer Graphics Proceedings (1994), Annual Conference Series, ACM Siggraph, pp. 303-310.

[16] Lounsbery,M., DeRose, T. D., and Warren, J. Multiresolution surfaces of arbitrary topological type. Department of Computer Science and Engineering 93-10-05, University of Washington, October 1993. Updated version available as 93-10-05b, January, 1994.

[17] Nasri, A. H. Surface interpolation on irregular networks with normal conditions. Computer Aided Geometric Design 8 (1991), 89-96.

[18] Peters, J. $C^{1}$ surface splines. SIAM J. Numer. Anal. 32, 2 (1995), 645-666.

[19] Peters, J. Curvature continuous spline surfaces over irregular meshes. Computer Aided Geometric Design (to appear).

[20] Reif, U. A unified approach to subdivision algorithms near extraordinary points. Computer Aided Geometric Design 12 (1995), 153-174.

[21] Sabin, M. The use of Piecewise Forms for the Numerical Representation of Shape. $\mathrm{PhD}$ thesis, Hungarian Academy of Sciences, Budapest, 1976.

[22] Schröder, P., and Sweldens, W. Spherical wavelets: Efficiently representing functions on the sphere. In Computer Graphics Proceedings (1995), Annual Conference Series, ACM Siggraph, pp. 161-172.

[23] Sweldens, W. The lifting scheme: A construction of second generation wavelets. Tech. Rep. 1995:06, Industrial Mathematics Initiative, Department of Mathematics, University of South Carolina, 1995.

[24] Warren, J. Subdivision methods for geometric design. Unpublished manuscript, November 1995.
[25] Zorin, D., Schröder, P., and Sweldens, W. Interpolating subdivision for meshes of arbitrary topology. Tech. Rep. CS-TR-96-06, Caltech, Department of Computer Science, Caltech, 1996. 